\title{
On Steady-State Voltage Stability Analysis Performance in MATLAB Environment
}

\author{
Jan Veleba \\ Regional Innovation Centre of Electrical Engineering \\ University of West Bohemia in Pilsen \\ Pilsen, Czech Republic \\ jveleba@rice.zcu.cz
}

\author{
Tomáš Nestorovič \\ New Technologies for the Information Society \\ University of West Bohemia in Pilsen \\ Pilsen, Czech Republic \\ nestorovic@ntis.zcu.cz
}

\begin{abstract}
Nowadays, electric power systems have been often operated close to their limits due to increased electric power consumptions, vast installments of renewable power sources and deliberated power market policies. This poses a serious threat to stable network operation and control. Therefore, voltage stability is currently one of key topics worldwide for preventing related black-out and islanding scenarios. In this paper, modelling and simulations of steady-state voltage stability problems in MATLAB environment are performed using author-developed computational tool implementing both conventional and more advanced numerical approaches. Their performance is compared with Simulink-based library Power System Analysis Toolbox (PSAT) in terms of solution accuracy, CPU time, and possible limitations. Their use for both real-time and off-line monitoring and assessment of system's voltage stability are also discussed.
\end{abstract}

Keywords-steady-state voltage stability; continuation load flow analysis; predictor-corrector method; voltage stability margin; voltage-power sensitivity; Power System Analysis Toolbox

\section{INTRODUCTION}

Steady-state voltage stability is defined as the capability of the system to withstand a small disturbance (e.g. fault occurrence, small change in parameters, topology modification, etc.) without abandoning a stable operating point [1]-[5]. Voltage stability problems are generally bound with long "electrical" distances between reactive power sources and loads, low source voltages, severe changes in system topology, and low level of var compensation. However, this does not strictly mean that voltage instability is directly connected only with low voltage scenarios. Voltage collapse can arise even during normal operating conditions (e.g. for voltages above nominal values). Moreover, variety of practical situations can eventually lead to voltage collapse, e.g. tripping of parallelly connected line during the fault, reaching the var limit of a generator or a synchronous condenser, restoring low supply voltage in induction motors after the fault. All of these cause the reduction of delivered reactive power for supporting bus voltages followed by increases of branch currents and further voltage drops to even lower reactive power flow or line tripping until the voltage collapse occurs. This entire process may appear in time from seconds to even tens of minutes.

To prevent voltage collapse scenarios, several types of compensation devices are massively used worldwide - both shunt capacitors/inductors, series capacitors, SVCs, synchronous condensers, STATCOMs, etc. To reduce voltage profiles (in case of low demand), var consumptions must be increased by switching in shunt reactors, disconnecting cable lines (if possible), reducing voltage-independent MVAr outputs from generators and synchronous condensers, etc. To increase bus voltages, opposite corrective actions are to be taken. These include reconfigurations (connecting parallel lines/cables/ transformers), power transfer limitations, and activations of new generating units at most critical network areas. Furthermore, under voltage load shedding of low-priority loads (usually by 5,10 or $20 \%$ in total) is usually realized at subtransmission substations using undervoltage relays. These relays work similarly as on-load tap-changing (OLTC) transformers. They are activated by long-term voltage dips (in region between 0.8 and $0.9 \mathrm{pu}$ ) and as the result, they trip the load feeders - typically in steps of 1 to $2 \%$ of the load at any given time (with time delays of 1-2 minutes after the voltage dip). The larger voltage dip, the faster and larger response of the relay [2].

Low voltage profiles are usually averted by actions of OLTC transformers. However, each tap position corresponds to an increase of the load which eventually leads to higher branch losses and further voltage drops [1]-[2],[4]. Therefore, OLTC transformers must be blocked during low voltage stability events. Negative effects of OLTC actions during low voltage conditions are presented in many studies with voltage stability margin calculations from synchrophasor measurements [6]-[7].

The rest of the paper is organized as follows. Sections II and III describe conventional Cycled Newton-Raphson (N-R) and more robust Continuation Load Flow (CLF) methods for voltage stability analysis, respectively. Independent tool Power System Analysis Toolbox (PSAT) - is briefly introduced in Section IV. In Section V, key properties of both of authordeveloped codes are discussed. Sections VI and VII show the results of all approaches when solving broad variety of test power systems. Finally, Section VIII evaluates each of the techniques applied, and concludes with planned future work.

\section{Conventional Numerical CAlculation OF The VOLTAGE STABILITY PROBLEM}

When increasing the loading (or loadability factor $\lambda$ ) of the system, its bus voltages slowly decrease due to the lack of reactive power. At the singular point, characterized by maximum loadability factor $\lambda_{\max }$ and critical bus voltages, the 
system starts to be unstable and voltage collapse appears. From this point on, only lower loading with low voltage values lead to the solution. The dependence between bus voltage magnitudes and $\lambda$ is graphically represented by the V-P curve, sometimes also referred to as the nose curve. Unfortunately, initial (base-case) position of the system operating point on the $\mathrm{V}-\mathrm{P}$ curve is not known along with its distance from the voltage collapse (i.e. voltage stability margin). Thus, location of the singular point must be found during the analysis.

Note: Values of $\lambda_{\max }$ and critical voltages are rather theoretical since they do not reflect voltage/flow limits of network buses/branches. When incorporating these practical restrictions, the real maximum loadability $\lambda_{\max }{ }^{*}$ can be found for keeping all bus voltages and branch loadings within limits.

The traditional approach for finding the maximum system loadability is to apply the standard N-R method [8] for the base-case load flow calculation (i.e. for $\lambda=1.0$ ). When obtaining current position on the V-P curve, network loading (i.e. loads/generations in selected network buses) is increased in defined manner by a certain step and the load flow is computed repetitively along with a new position on the V-P curve. This process continues in an infinite loop until the singular point is reached. However, total number of iterations in each V-P step is gradually increasing so that when close to the singular point, the N-R method fails to converge, i.e. no solution is provided. This relates to the fact that Jacobian $\underline{J}$ becomes singular (i.e. det $\underline{J} \approx 0$ ) and its inverse matrix cannot be computed for successful numerical convergence.

To speed up the calculation, variable step change is applied. Usually, a single step value is used. When obtaining the divergence of the N-R method, the step size is simply divided by two and the calculation for the current V-P point is repeated until the convergence is achieved. When the current step size value reaches the predefined minimum value, the calculation is stopped. Despite of the relatively simple procedure, Cycled N$\mathrm{R}$ method enables the completion of the stable V-P curve only. Unstable part including the singular point cannot be examined. Also, high CPU requirements prevent this method from being employed for larger power systems.

In this paper, Cycled N-R algorithm was developed and further tested on wide range of test power systems.

\section{CONTINUATION LOAD FlOW ANALYSIS}

CLF analysis [1],[9] suitably modifies conventional load flow equations to become stable also in the singular point. Eventually, both upper/lower parts of the V-P curve can be drawn. It uses a two-step predictor/corrector algorithm along with the new unknown state variable called continuation parameter (CP). Predictor (1) is a tangent extrapolation of the current operation point estimating approximate position of the new point on the V-P curve.

$$
\left[\begin{array}{c}
\theta \\
V \\
\lambda
\end{array}\right]^{\text {predicted }}=\left[\begin{array}{c}
\theta_{0} \\
V_{0} \\
\lambda_{0}
\end{array}\right]+\sigma\left[\begin{array}{cccc} 
& \vdots & \\
& \underline{J} & \vdots & K \\
& & \vdots & \\
\cdots & \ldots & \ldots & \ldots \\
& & e_{k} &
\end{array}\right]^{-1}\left[\begin{array}{c}
0 \\
\vdots \\
0 \\
1
\end{array}\right]
$$

Vector $K$ contains base-case power generations and loads. Variables $\theta_{0}, V_{0}, \lambda_{0}$ define the system state from the previous corrector step. Vector $e_{k}$ is filled with zeros and certain modifications (see [1],[9]) are implemented for selected CP in each network bus $k$ at the current point on the V-P curve. Remaining elements in (1) are the newly computed Jacobian $\underline{J}$ and step size $\sigma$ of the CP.

Tangent predictor is relatively slow, anyway shows good behaviour especially in steep parts of the V-P curve. Unlike tangent predictor, secant predictor is simpler, computationally faster and behaves well in flat parts of the V-P curve. In steep parts (i.e. close to the singular point and at sharp corners when a generator exceeds its var limit) it computes new predictions too far from the exact solution. This may eventually lead to serious convergence problems in the next corrector step. Thus, tangent predictor is more recommended to be applied.

Corrector is a standard N-R algorithm for correcting state variables from the predictor step to satisfy load flow equations. Due to one extra parameter $\lambda$, additional condition (2) must be included for keeping the value of the $\mathrm{CP}$ constant in the current corrector step. This condition makes the final set of equations non-singular even at the bifurcation point.

$$
x_{k}-x_{k}^{\text {predicted }}=0, x= \begin{cases}\lambda & \text { if } \mathrm{CP} \text { is } \lambda \\ V & \text { if } \mathrm{CP} \text { is } V\end{cases}
$$

For the $\mathrm{CP}$, state variable with the highest rate of change must be chosen (i.e. $\lambda$ and $V$ in flat and steep parts of the V-P curve, respectively). If the process diverges, parameter $\sigma$ must be halved or parameter CP switched from $\lambda$ to $V$.

Difference between both types of predictors and the entire process of the predictor/corrector algorithm is demonstrated in Fig. 1. Horizontal/vertical corrections are performed with respect to chosen $\mathrm{CP}$ type.
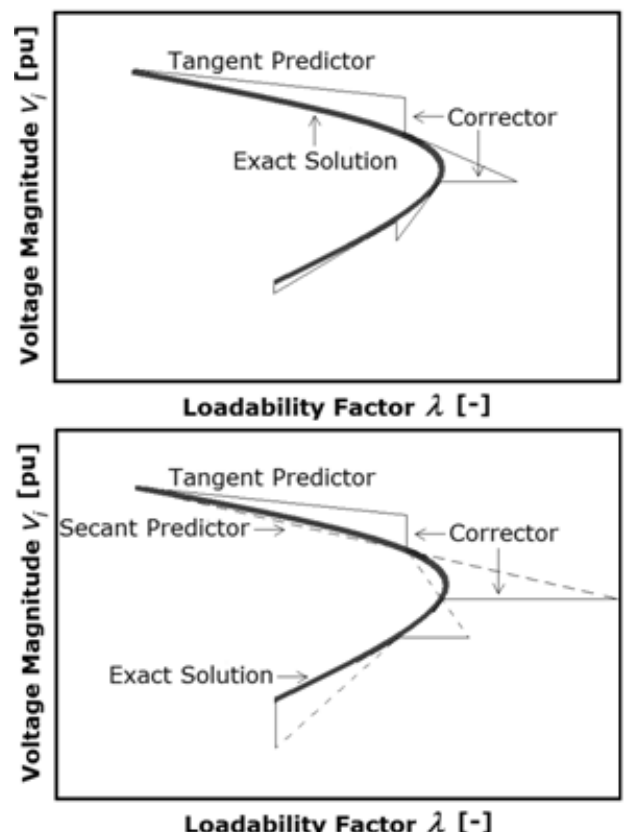

Fig. 1. Predictor/Corrector Mechanism for CLF Analysis [10]. 
Step size should be carefully increased to speed up the calculation when far from the singular point, or decreased to avoid convergence problems when close to the peak. Step size modification based on the current position on the V-P curve (i.e. as a function of the line slope for previous two corrected points on the V-P curve) is recommended in [11]. This approach belongs to so-called rule-based or adaptive step size control algorithms.

In [10], several voltage stability margin indices (VSMI, $\mathrm{VSMI}_{i k}$ ) are presented along with relative var reserve coefficient and voltage-load sensitivity factors $\left(\mathrm{VSF}_{i}\right)$ for comprehensive voltage stability analysis and location of weak or sensitive system buses/branches/areas. In these regions, preventive or remedial actions should be taken. Procedures for allocating individual compensation devices and possible effects are also discussed.

CLF analysis still remains very popular for high-speed solving of voltage stability studies. Due to its reliable numerical behaviour, it is often included into the N-R method providing stable solutions even for ill-conditioned load flow cases. Moreover, it is applied in foreign control centres for $\mathrm{N}-1$ on-/off-line contingency studies with frequencies of 5 and 60 minutes [2], respectively.

\section{POWER SYSTEM ANALYSIS TOOLBOX (PSAT)}

PSAT [12] is a Simulink-based open-source library for electric power system analyses and simulations, distributed via General Public License (GPL). It contains the tools for Power Flow (busbars, lines, two-/three-winding transformers, slack bus(es), shunt admittances, etc.), CLF and OPF data (power supply/demand bids and limits, generator power reserves and ramping data), Small Signal Stability Analysis and Time Domain Simulations. Moreover, line faults and breakers, various load types, machines, controls, OLTC transformers, FACTS and other can be also modelled. User defined device models can be added as well.

All studies must be formulated for one-line network diagram only - either in input data *.m file in required format or in graphical *.mdl file containing manually drawn network scheme. For the former option, input data conversions from and to various common formats are available (e.g. PSS/E, DIgSILENT, IEEE cdf, NEPLAN, PowerWorld and others).

When compared to another MATLAB-based open-source tool MATPOWER [13], PSAT is more efficient and highly advanced by providing more analyses, problem variations, possible outputs and other useful features in its user-friendly graphical interface. MATPOWER does not support most of advanced network devices, entirely omits CLF analysis and has neither graphical user interface nor graphical network construction ability. Also, it does not consider var limits in PV buses completely. Incorrect interpretation of reactive power branch losses can be also observed.

\section{PROPERTIES OF AUTHOR-DEVELOPED CODES IN MATLAB ENVIRONMENT}

As shown in the previous section, there exists a MATLABbased aplication that meets the requirements for steady-state voltage stability analysis of electric power systems. Due to several limitations and CPU time restrictions (see Section VII), our goal was to focus primarily on speed/precision impromevements of voltage stability studies. Therefore, a specialized tool in MATLAB environment was developed using both Cycled N-R and CLF routines for providing fundamental examination of medium-sized and larger power systems in terms of steady-state voltage stability. Several key aspects of these codes are discussed below.

1] Predictor: Despite of computationally more expensive algorithm, tangent predictor was used for finding reliable estimations of new V-P points especially around the singular point. Applied in CLF algorithm only.

2] Corrector: First, corrector step is used at the start of the CLF program to find the base-case point for further calculations. Due to possible weak numerical stability at this point (for badly-scaled power systems), the One-Shot FastDecoupled (OSFD) procedure is implemented to the standard $\mathrm{N}-\mathrm{R}$ method for providing more stable solutions and thus preventing numerical divergence. Moreover, voltage truncation (SUT algorithm) is also included into the state update process at every N-R's iteration. Both of these stability approaches were introduced in [3] and further tuned and tested in [14]. Both were also applied to Cycled N-R algorithm to increase the loading range for which the stable load flow solutions can be obtained. Thus, closer proximity to singular point can be reached.

3] Step size: Largest-load PQ network bus is chosen for computing the angle $\alpha$ between the horizontal and the line interconnecting two adjacent V-P points. Based on this, step size evaluation function (3) is applied - see Fig. 2.

$$
\sigma=\left\{\begin{array}{ccc}
\sigma_{\mathrm{L}} & \text { for } & |\alpha| \geq \pi / 8 \\
\sigma_{\mathrm{U}} & \text { for } & |\alpha| \leq \pi / 32 \\
A / \sin ^{2} \alpha+B & & \text { otherwise }
\end{array}\right.
$$

The upper and lower step limit constants $\sigma_{\mathrm{U}}$ and $\sigma_{\mathrm{L}}$ define the step size for the flat part of the V-P curve and for close vicinity to the singular point, respectively.

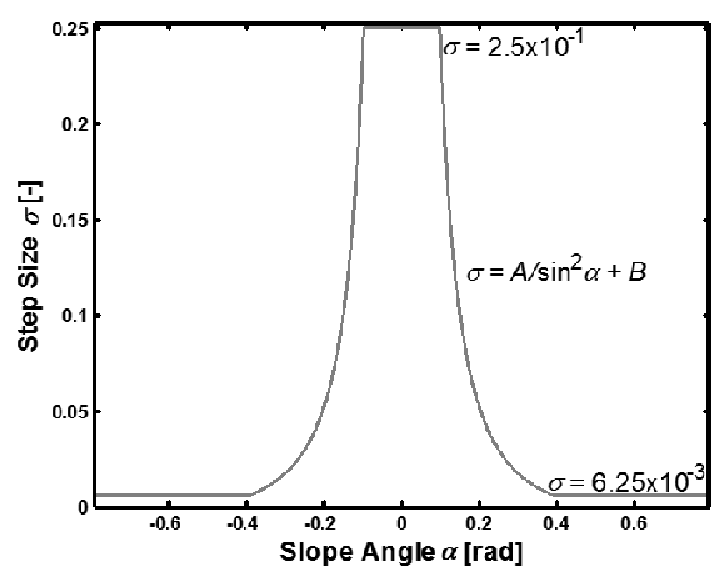

Fig. 2. Step size evaluation function [10].

For Cycled N-R algorithm, this is a rather too complex concept of step size control. Therefore, only a single step size 
is chosen at the start and a simple step-cutting technique (dividing by 2) is applied in case of divergence.

4] Ending criterion: Only stable part of the V-P curve (incl. exact singular point calculation) is computed by CLF code. Thus, if the computed value of $\lambda$ begins to decrease, the process is stopped. For Cycled N-R code, the calculation is terminated when the step size falls below a given small value (e.g. $1 \times 10^{-8}$ ). For each load flow case, maximum number of iterations and permitted tolerance for convergence is set to 20 and $1 \times 10^{-8}$, respectively.

5] Calculation speed and accuracy: For excessively accurate voltage stability solutions, values of $2.5 \times 10^{-2}$ and $6.25 \times 10^{-4}$ are used for constants $\sigma_{\mathrm{U}}$ and $\sigma_{\mathrm{L}}$ in CLF algorithm. Rather compromise values of $5 \times 10^{-2}$ and $1 \times 10^{-2}$ are also used to obtain fast and fairly accurate solutions for any of tested power systems. For Cycled N-R algorithm, initial step size of $2.5 \times 10^{-2}$ seems to be sufficient enough.

6] Code versatility: Both Cycled N-R and CLF procedures are programmed so that the user can directly specify an arbitrary group of network buses for load/generation increase. From this set of buses, only those non-slack buses with nonzero active power loads/generations are involved into the analysis. In each of studies performed, load/generation increase in the entire network was considered, i.e. all network buses were selected.

Two scenarios can be activated by the user. a) L scenario increases both $\mathrm{P} / \mathrm{Q}$ loads in selected $\mathrm{PQ} / \mathrm{PV}$ buses with constant power factor (i.e. with identical increase rate). b) $\mathrm{L}+\mathrm{G}$ scenario increases both $\mathrm{P} / \mathrm{Q}$ loads in selected $\mathrm{PQ} / \mathrm{PV}$ buses and $\mathrm{P}$ generations in selected PV buses (with identical increase rate).

7] Var limits: In both approaches, bus-type switching logics are applied to iteratively computed reactive powers $Q_{G i}$ in PV buses when exceeding the var limit (4), or to relevant bus voltages when returning the vars back inside the permitted var region (5).

$$
\begin{gathered}
Q_{G i}=\left\{\begin{array}{lll}
Q_{G i \max } & \text { if } & Q_{G i}>Q_{G i \max } \\
Q_{G i \min } & \text { if } & Q_{G i}<Q_{G i \min }
\end{array}\right. \\
V_{i}=V_{i}^{s p} \text { if }\left\{\begin{array}{c}
Q_{G i}=Q_{G i \max } \text { AND } V_{i}>V_{i}^{s p} \\
\text { OR } \\
Q_{G i}=Q_{G i \min } \text { AND } V_{i}<V_{i}^{s p}
\end{array}\right\}
\end{gathered}
$$

Variables $Q_{G i \max }$ and $Q_{G i \text { min }}$ are the upper and lower var limits, while $V_{i}^{s p}$ determines the specified value of voltage magnitude for each PV bus.

8] Code limitations: a) With increased loading, lower/upper var limits in PV buses should not be fixed but vary proportionally to the generated active power. In both codes, constant var limits are used for more pessimistic V/Q control. b) Only identical increase rate is applied. However, implementing user-defined increase rates for each generation/ load would not pose any serious problems.

9] Outputs: Theoretical value of $\lambda_{\max }$ and $V-\lambda$ data outputs for V-P curves are computed and stored or graphically projected. Respective values of $\lambda$ for switching some of PV buses permanently to PQ are also recorded. Voltage and power flow limits were not considered for the evaluation of real maximum loadability $\lambda_{\max }{ }^{*}$.

10] Sparse programming: Sparsity techniques along with smart vector/matrix programming are used in both Cycled N-R and CLF codes to significantly decrease the CPU time needed for each load flow case.

\section{TESTING CYCLED N-R AND CLF ALGORITHMS FOR Solving Voltage Stability LoAd Flow Problems}

Total number of 50 test power systems between 3 and 734 buses were analyzed using developed Cycled N-R and CLF algorithms in MATLAB environment. Identical increase rate was applied to all network buses (before filtering those with non-zero active power loads or generations). For both $\mathrm{L}$ and $\mathrm{L}+\mathrm{G}$ scenarios, only stable part of the V-P curve was calculated with var limits included. Settings of both codes are as introduced in Section V, Paragraphs 4 and 5. In Table 1, voltage stability solutions of several test cases are shown. Presented results contain the maximum loadability, numbers of stable V-P points and CPU times in seconds needed. For each of the cases, the first two rows show the outputs of the CLF code for excessive and compromise accuracy, respectively. As the comparison, the third row provides the results of Cycled $\mathrm{N}$ $\mathrm{R}$ code.

As it can be seen, exact solutions of maximum loadability were obtained for both of tested methods and each of the three accuracy settings. The first setting was apparently too much focused on producing exact results. Therefore, numbers of V-P points and CPU times often exceeded 200 and 1 second, respectively. When using fair compromise setting, the maximum error for $\lambda_{\max }$ from all 50 test power systems was only 0.0185 percent, while numbers of points and CPU times were decreased on average by 75.27 percent and 64.11 percent, respectively.

TABLE I. VOLTAGE STABILITY SOLUTIONS USING CYCLED N-R AND

\begin{tabular}{|c|c|c|c|c|c|c|}
\hline \multirow{2}{*}{ Case } & \multicolumn{3}{|c|}{ Scenario L } & \multicolumn{3}{|c|}{ Scenario $\mathrm{L}+\mathrm{G}$} \\
\hline & $\lambda_{\max }[-]$ & points & time [s] & $\lambda_{\max }[-]$ & points & time $[\mathrm{s}]$ \\
\hline \multirow{3}{*}{ IEEE9 } & 1.302632 & 331 & 0.5616 & 1.162053 & 215 & 0.3900 \\
\hline & 1.302632 & 27 & 0.1404 & 1.162052 & 24 & 0.1248 \\
\hline & 1.302632 & 23 & 0.4056 & 1.162053 & 20 & 0.4212 \\
\hline \multirow{3}{*}{ IEEE14 } & 1.760331 & 658 & 1.2012 & 1.777995 & 506 & 0.9360 \\
\hline & 1.760331 & 87 & 0.2340 & 1.777995 & 59 & 0.2028 \\
\hline & 1.760331 & 43 & 0.5460 & 1.777995 & 45 & 0.6396 \\
\hline \multirow{3}{*}{ IEEE30 } & 1.536905 & 854 & 1.9500 & 1.546751 & 726 & 1.6536 \\
\hline & 1.536905 & 88 & 0.2808 & 1.546752 & 124 & 0.4212 \\
\hline & 1.536905 & 37 & 0.6396 & 1.546751 & 37 & 0.6552 \\
\hline \multirow{3}{*}{ IEEE57 } & 1.406778 & 891 & 2.9016 & 1.616845 & 399 & 1.3884 \\
\hline & 1.406778 & 229 & 0.6864 & 1.616845 & 57 & 0.2652 \\
\hline & 1.406778 & 27 & 0.8112 & 1.616845 & 37 & 0.8112 \\
\hline \multirow{3}{*}{ IEEE162 } & 1.079959 & 1640 & 12.9169 & 1.138996 & 1185 & 9.3913 \\
\hline & 1.079960 & 464 & 3.1044 & 1.138996 & 65 & 0.8112 \\
\hline & 1.079960 & 13 & 1.7628 & 1.138996 & 16 & 1.8408 \\
\hline \multirow{3}{*}{ IEEE300 } & 1.024573 & 8457 & 103.8655 & 1.058820 & 311 & 4.0092 \\
\hline & 1.024573 & 529 & 7.0044 & 1.058819 & 94 & 1.4508 \\
\hline & 1.024573 & 16 & 2.4180 & 1.058820 & 17 & 2.5584 \\
\hline \multirow{3}{*}{ EPS734 } & 3.104162 & 139 & 4.5864 & 3.104162 & 139 & 4.8360 \\
\hline & 3.104083 & 46 & 1.8720 & 3.104083 & 46 & 1.8408 \\
\hline & 3.104162 & 96 & 8.2369 & 3.104162 & 96 & 8.1745 \\
\hline
\end{tabular}
CLF ALGORITHMS - L AND L+G SCENARIOS 
Cycled N-R code obtains highly accurate results in terms of solution accuracy. In majority of cases, it provides even better solutions than CLF algorithm with compromise accuracy. Surprisingly, it always computes slightly higher maximum loadability values than the highly accurate CLF code. This seems to be one of visible drawbacks of Cycled N-R method. Only low numbers of V-P points are needed for reaching close proximity to the singular point. These numbers are well comparable to those needed for compromise CLF code. Unfortunately, each divergence case (between 22 and 28) significantly prolongs the entire computation process of Cycled $\mathrm{N}-\mathrm{R}$ method. Therefore, Cycled N-R code suffers from being extremely time-dependent on computing each V-P point. When compared to compromise CLF code, the CPU time needed by Cycled N-R method is on average about $167 \%$ higher. Therefore, compromise CLF code seems to be the best method for providing fast and highly accurate voltage stability results.

Stable V-P curves of the IEEE 30-bus power system ( $\mathrm{L}+\mathrm{G}$ scenario) were computed using both Cycled N-R and CLF methods, and they are shown in Figs. 3 and 4, respectively. For CLF method, the V-P curves are extended to demonstrate numerical stability of CLF algorithm around the singular point. Extension of V-P curves in the unstable region is provided for $0.97 \times \lambda_{\max }<\lambda<\lambda_{\max }$.

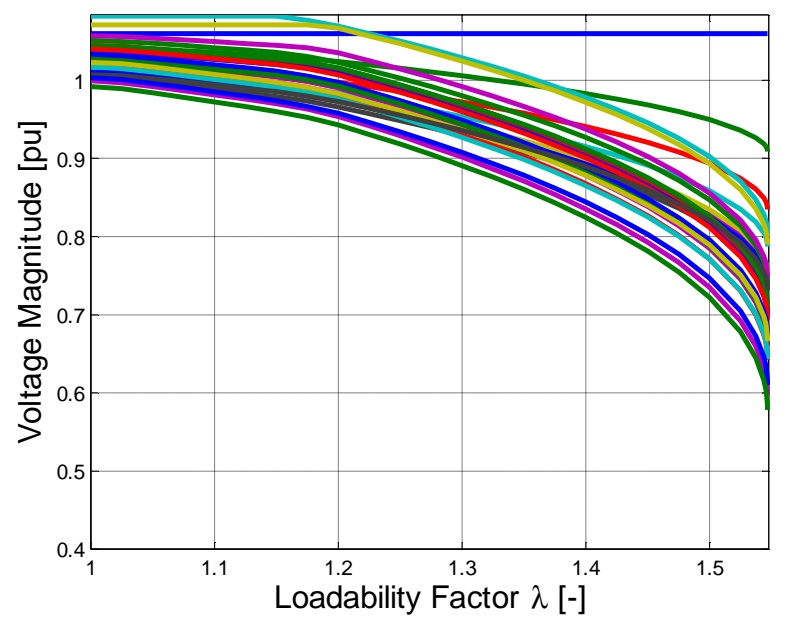

Fig. 3. V-P curves for the IEEE 30-bus system (Cycled N-R method).

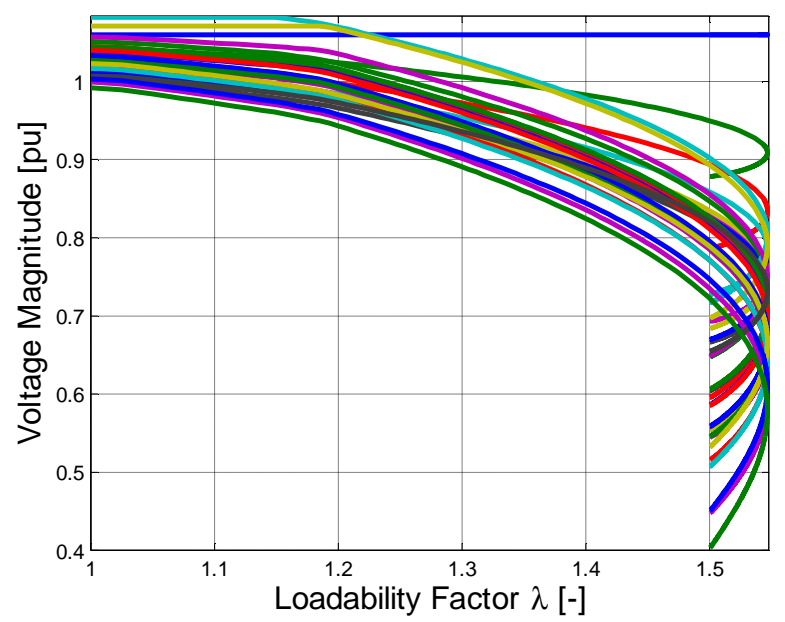

Fig. 4. Extended V-P curves for the IEEE 30-bus system (CLF method).
As Table 1 shows, applied version of CLF method is still not applicable for real-time voltage stability monitoring, but it can be useful for off-line reliability, evaluation or planning studies of even larger networks.

\section{Testing PSAT For SOlVING Voltage Stability LOAD FLOW PROBLEMS}

Despite PSAT has many advantages, it also suffers from several drawbacks. Four of them were spotted during the testing stage when large number of load flow studies was solved using PSAT and results were compared with authordeveloped N-R code in MATLAB environment. First, inefficient PV-PQ bus type switching logic is applied. Probably, reverse switching logic (5) is not used and the need for convergence is requested to activate forward switching logic (4). As a result, unnecessarily more PV buses are being switched permanently to PQ. Furthermore, switching logic completely fails to switch PV buses to PQ for larger systems with high numbers of PV buses. Second, nominal voltages must be defined in the input data file otherwise the error message 'Divergence - Singular Jacobian' is obtained during the simulation. This seems to be entirely illogical since nominal voltages should not be necessary for the 'in per units defined' problem. Third, it seems that no advanced stability techniques are applied for the N-R method in PSAT because of severe numerical oscillations appearing in several studies. Finally fourth, PSAT intentionally neglects transformer susceptances and thus causes errors in final load flow results. A column for shunt susceptances is available for power lines only. For transformers, this column is reset to zero automatically.

Under these limitations, load flow results show very good congruity between author-developed N-R method and PSAT. Higher total numbers of iterations are needed by PSAT due to missing stability technique(s). Also, CPU times are higher in PSAT due to combining the codes with other analyses and related tool features.

As an example, load flow and voltage stability analysis of the IEEE 14-bus system is accomplished by PSAT - see Figs. 5-9).

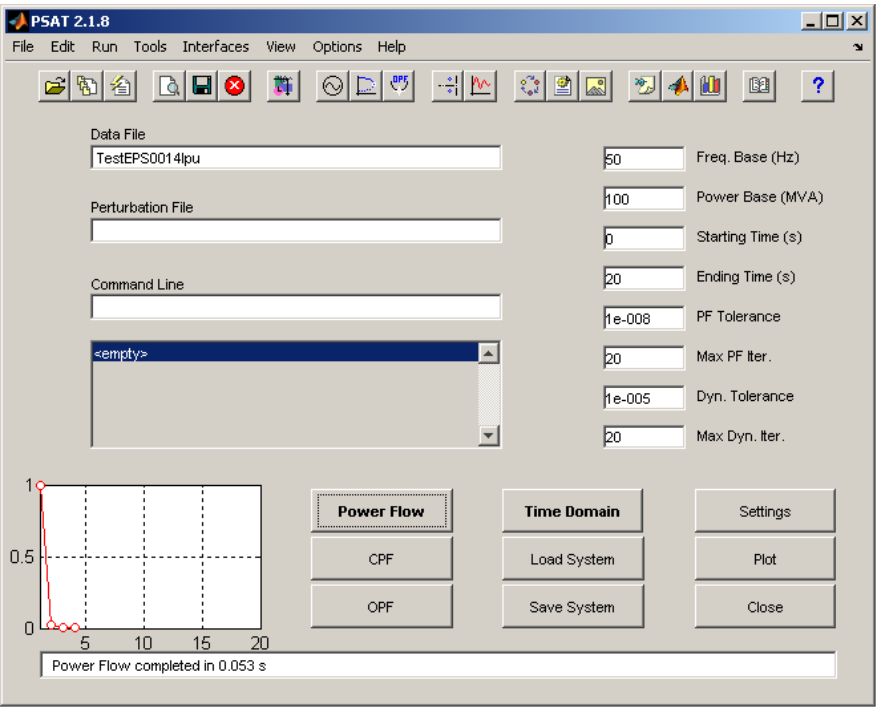

Fig. 5. GUI in PSAT for load flow analysis of IEEE 14-bus system. 


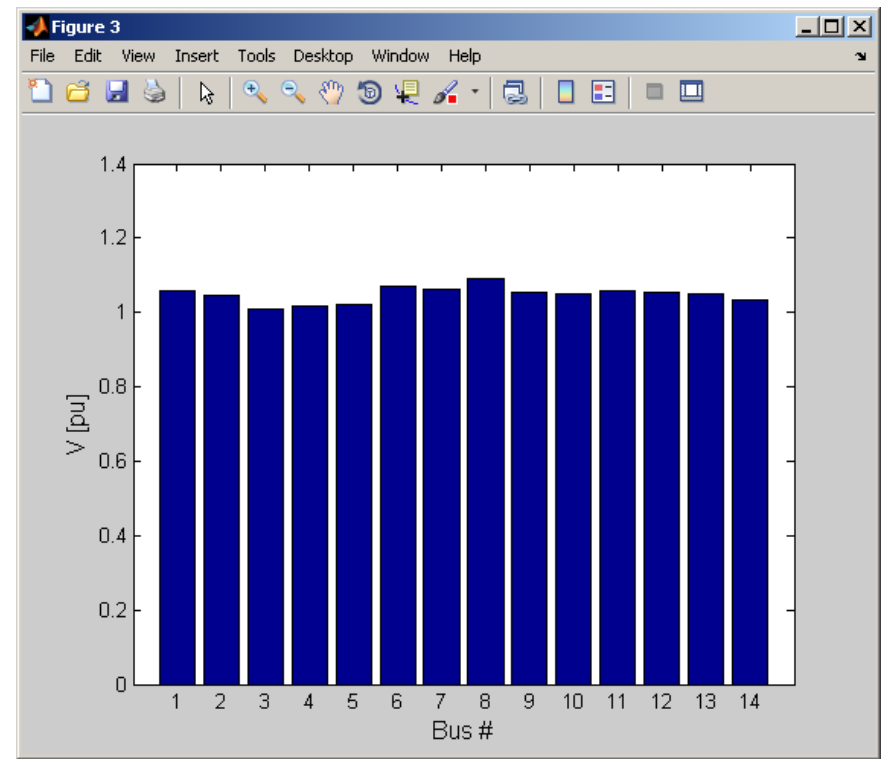

Fig. 6. Final voltage magnitudes of IEEE 14-bus power system in PSAT.

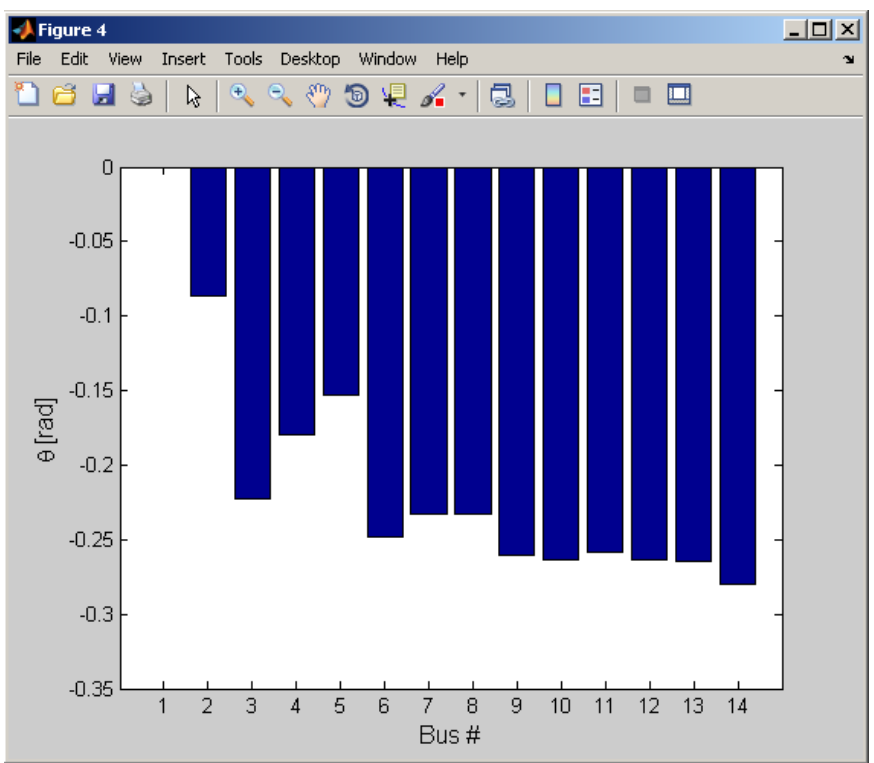

Fig. 7. Final voltage angles of IEEE 14-bus power system in PSAT.

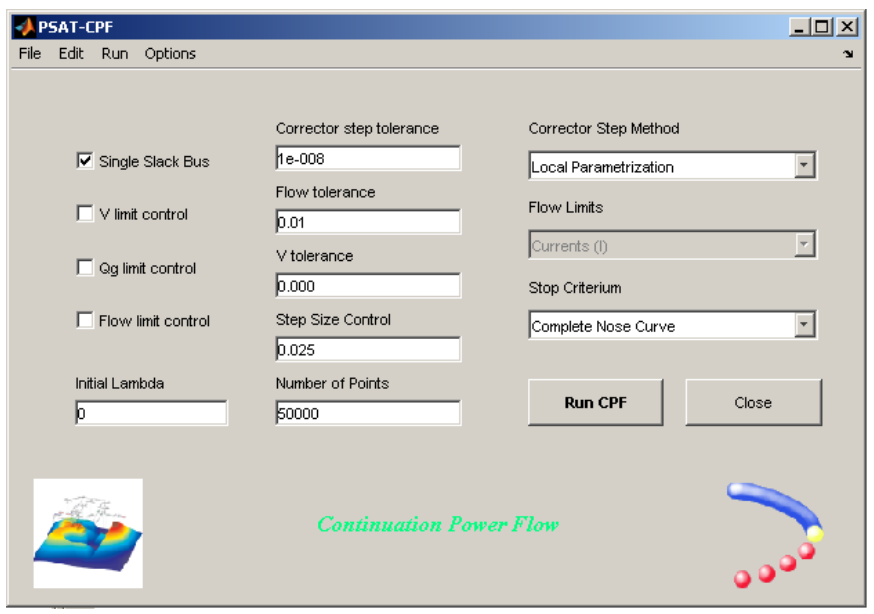

Fig. 8. Settings of CLF code for solving the IEEE 14-bus power system.

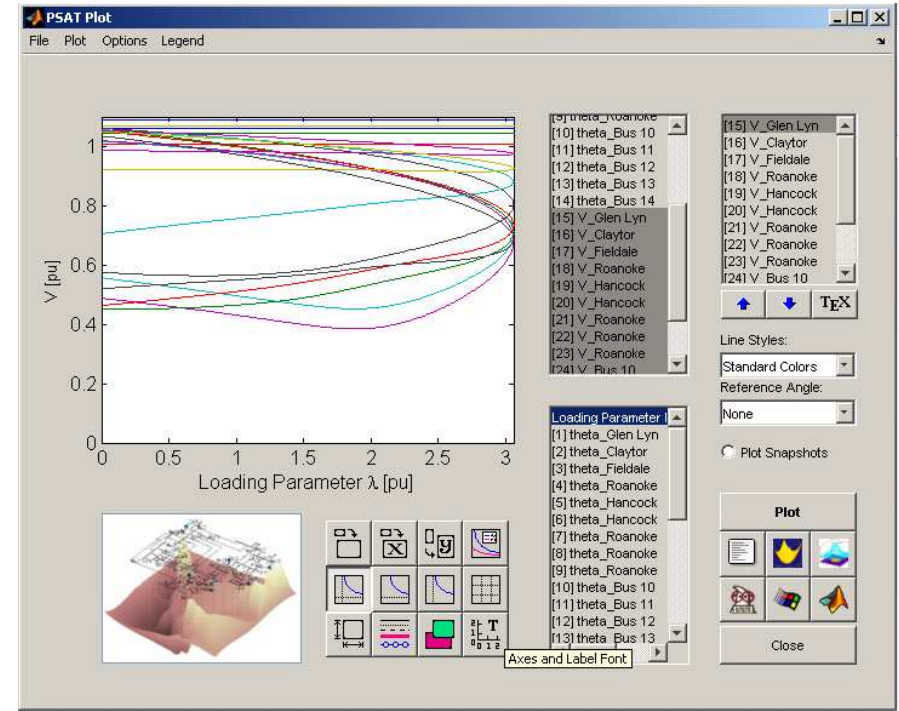

Fig. 9. Nose curves for all network buses of the IEEE 14-bus test system.

For voltage stability studies, PSAT contains the advanced CLF algorithm combining contingency and OPF analyses. Load flow data are extended by two matrices specifying the sets of $\mathrm{PQ} / \mathrm{PV}$ buses where the loads/generations are to be increased (different increase rates are possible). CLF code is then started via specialized window (Fig. 8). Calculation can be adjusted by the user for better computational performance - e.g. by setting more suitable step size, maximum number of $\mathrm{V}-\mathrm{P}$ points, or by checking the option for controlling voltage, flow or var limits. PSAT offers two CLF methods - perpendicular intersection (PI) and local parametrization (LP). Three stopping criteria are available: Complete Nose Curve (computing both stable/unstable parts of the V-P curve), Stop at Bifurcation (when singular point exceeded) and Stop at Limit (when voltage/flow/point limit reached).

CLF algorithm in PSAT is defined so that power increases are realized by adding a power increment (loadability factor multiplied by increase rate) to the base-case loading, i.e. initial $\lambda$ is zero. In author-developed Cycled N-R and CLF codes, power increases are performed by multiplying the base-case loading with $\lambda$. Therefore, maximum loadability in PSAT must be increased by unity when comparing both codes. In Table 2 , voltage stability solutions for medium-sized IEEE test systems are provided by the author-developed Cycled N-R and compromise CLF codes when compared to those obtained by PSAT (PI mode with step 0.025 and LP mode with default step $0.5)$. As the outputs, theoretical values of $\lambda_{\max }$, numbers of stable V-P points and CPU times were stored. For all voltage stability studies in PSAT, identical power increase rates $(\mathrm{L}+\mathrm{G}$ scenario) were applied with deactivated logics for var limits.

Both of PSAT modes showed only average accuracy with satisfiable numbers of V-P points and lower computing speed. LP mode was more time-consuming, but needed lower numbers of V-P points and usually provided more accurate results. Compromise CLF code provided the best combination of solution accuracy and CPU times in each of the cases. Although higher numbers of V-P points were needed, CPU times were still rather smaller than those in PSAT due to optimized sparse programming applied. Identical conclusions can be made when mutually comparing CLF and Cycled N-R codes. 
TABLE II. VOLTAGE STABILITY ANALYSIS OF MEDIUM-SIZED IEEE TEST SYSTEMS (AUTHOR-DEVELOPED VS. PSAT ROUTINES)

\begin{tabular}{|c|c|c|c|c|c|c|c|c|c|c|c|c|}
\hline \multirow{3}{*}{ Case } & \multicolumn{6}{|c|}{ Author-developed routines } & \multicolumn{6}{|c|}{ PSAT routines } \\
\hline & \multicolumn{3}{|c|}{ Cycled N-R code } & \multicolumn{3}{|c|}{ Compromise CLF code } & \multicolumn{3}{|c|}{ PSAT - PI mode } & \multicolumn{3}{|c|}{ PSAT - LP mode } \\
\hline & $\lambda_{\max }[-]$ & points & time [s] & $\lambda_{\max }[-]$ & points & time [s] & $\lambda_{\max }[-]$ & points & time $[\mathrm{s}]$ & $\lambda_{\max }[-]$ & points & time $[\mathrm{s}]$ \\
\hline IEEE9 & 2.485393 & 74 & 0.5460 & 2.485382 & 84 & 0.2964 & 2.481220 & 7 & 0.2093 & 2.482000 & 13 & 0.3241 \\
\hline IEEE13 & 4.400579 & 148 & 0.6708 & 4.400577 & 112 & 0.3120 & 4.390420 & 13 & 0.3292 & 4.399570 & 20 & 832 \\
\hline IEEE14 & 4.060253 & 137 & 0.8268 & 4.060252 & 92 & 0.3276 & 4.060100 & 18 & 0.4098 & 4.059420 & 19 & 0.4939 \\
\hline IEEE24 & 2.279398 & 61 & 0.6396 & 2.279398 & 58 & 0.2496 & 2.277550 & 10 & 0.2600 & 2.278670 & 16 & 0.4313 \\
\hline IEEE30 & 2.958815 & 88 & 0.8112 & 2.958814 & 57 & 0.2964 & 2.958550 & 16 & 0.8761 & 2.958250 & 20 & 1.5023 \\
\hline IEEE35 & 2.888962 & 91 & 0.6864 & 2.888950 & 107 & 0.3432 & 2.872940 & 16 & 1.1242 & 2.878420 & 10 & 0.2940 \\
\hline IEEE39 & 1.999203 & 57 & 0.7644 & 1.999202 & 30 & 0.2184 & 1.999110 & 11 & 0.2932 & 1.997840 & 12 & 0.3692 \\
\hline IEEE57 & 1.892091 & 47 & 0.8892 & 1.892089 & 92 & 0.4836 & 1.891920 & 12 & 0.9089 & 1.892090 & 26 & 3.9090 \\
\hline IEEE118 & 3.187128 & 100 & 1.6536 & 3.187128 & 66 & 0.5772 & 3.187100 & 613 & 19.1693 & 3.187120 & 82 & 19.7464 \\
\hline
\end{tabular}

\section{CONCLUSION}

For solving voltage stability problems, both Cycled N-R and CLF codes were implemented and thoroughly tested on a broad range of test power systems in MATLAB environment. Various stability techniques, step size approaches and numerical settings were applied and used to upgrade their performance in order to find the algorithm with fair compromise between calculation speed and solution accuracy. Their final results were compared to outputs obtained from PSAT. The studies imply that the technique with the best combination of precision level and CPU time requirements is the CLF algorithm with compromise step size settings.

However, the resulting technique is the best to be used for off-line planning and development studies of electric power systems only. For real-time evaluations of system's voltage stability, more robust algorithms with minimized numbers of stable V-P points are to be developed. Therefore, follow-up research activities will be concentrated especially on this area of interest.

\section{REFERENCES}

[1] P. Kundur, Power System Stability and Control. McGraw-Hill, 1994.

[2] C. Canizares, A.J. Conejo and A.G. Exposito, Electric Energy Systems: Analysis and Operation. CRC Press, 2008.
[3] V. Ajjarapu, Computational Techniques for Voltage Stability Assessment and Control. Springer, 2006.

[4] I. Dobson, T.V. Cutsem, C. Vournas, C.L. DeMarco, M. Venkatasubramanian, T. Overbye and C.A. Canizares, "Voltage Stability Assessment: Concepts, Practices and Tools," IEEE-PES, 2002.

[5] J.H. Chow, F.F. Wu and J.A. Momoh, Applied Mathematics for Restructured Electric Power Systems - Optimization, Control and Computational Intelligence. Springer, 2005.

[6] I. Šmon, M. Pantoš and F. Gubina, "An improved voltage-collapse protection algorithm based on local phasors," Electric Power Systems Research 78 (2008), Vol. 78, 2008, pp. 434-440.

[7] Y. Gong and N. Schulz, "Synchrophasor-Based Real-Time Voltage Stability Index," Proceedings of PSCE conference, 2006, pp. 1029-1036.

[8] J.J. Grainger and W.D. Stevenson, Power System Analysis. McGrawHill, 1994.

[9] M. Crow, Computational Methods for Electric Power Systems. CRC Press, 2002.

[10]J. Veleba, "Application of Continuation Load Flow Analysis for Voltage Collapse Prevention,” Journal Acta Technica, Vol. 57, 2012, pp. 143-163.

[11]P. Zhu, Performance Investigation of Voltage Stability Analysis Methods, $\mathrm{PhD}$. thesis. Brunel University of West London, 2008.

[12]Homepage of PSAT. [Online]. Available: http://www3.uclm.es/ profesorado/federico.milano/psat.htm. [Accessed: 3 Mar. 2013].

[13] Homepage of MATPOWER. [Online]. Available: www.pserc.cornell.edu/ matpower/. [Accessed: 23 Dec. 2012].

[14] J. Veleba, "Acceleration and Stability Techniques for Conventional Numerical Methods in Load Flow Analysis," Proceedings of Elekroenergetika (ELEN) conference, 2010, pp. 1-10.

\section{Creative Commons Attribution License 4.0 (Attribution 4.0 International, CC BY 4.0)}

This article is published under the terms of the Creative Commons Attribution License 4.0

https://creativecommons.org/licenses/by/4.0/deed.en_US 